\title{
RIBONUCLEASES. POSSIBLE NEW APPROACH IN CANCER THERAPY
}

\author{
V.O. Shlyakhovenko \\ R.E. Kavetsky Institute of Experimental Pathology, Oncology and Radiobiology, NASof Ukraine, Kyiv 03022, Ukraine
}

In the review, the use of the ribonucleases for cancer therapy is discussed. Using of epigenetic mechanisms of regulation - blocking protein synthesis without affecting the DNA structure - is a promising direction in the therapy. The ribonucleases isolated from different sources, despite of similar mechanism of enzymatic reactions, have different biological effects. The use of enzymes isolated from new sources, particularly from plants and fungi, shows promising results. In this article we discuss the new approach for the use of enzymes resistant to inhibitors and ribozymes, that is aimed at the destruction of the oncogene specific mRNA and the induction of apoptosis. Key Words: RNases, RNA cleavage, cytotoxicity, apoptosis, cancer therapy.

Ribonucleases (RNases) are a large group of hydrolytic enzymes that degrade ribonucleic acid (RNA) molecules. They are nucleases that catalyze the breakdown of RNA into smaller components. RNases are a superfamily of enzymes which catalyzing the degradation of RNA operate at the levels of transcription and translation. They can be cytotoxic because the cleavage of RNA renders illegible its information. Initially it was assumed that the function of these enzymes is a simple destruction of polyribonucleotides already fulfilled their physiological role. Later, however, it became clear that the RNases present in all living cells and biological fluids, like pro- and eukaryotes, perform many important vital functions. Some members of this family possess a high molecular heterogeneity, with some isoforms that exhibit extremely high specificity for cleavage of specific loci in the polyribonucleotide chains.

RNases are ubiquitous, with a very short lifespan in an unprotected environment. The cytotoxic effects of RNases include the RNA cleavage leading to the inhibition of the protein synthesis and inducing apoptosis [1]. The cytotoxic effect can be produced by applying RNase on the outside surface of the cell but it was found that cytotoxicity increases about by 1000 times when enzyme is artificially introduced into the cytosol, indicating internalization into the cell as the rate limiting step for toxicity [2]. Mi et al. [3] showed evidence that RNASEL Arg462GIn and Asp$541 \mathrm{Glu}$ polymorphisms are associated with prostate cancer risk and could be low-penetrance prostate cancer susceptibility biomarkers.

There are two types of RNases, endoribonucleases and exoribonucleases. Endoribonucleases are RNase A, RNase P, RNase H, RNase III, RNase T1, RNase T2, RNase U2, RNase V1, RNase I, RNase PhyM and RNase V. Exoribonucleases includes

Submitted: September 30, 2015.

Correspondence: E-mail: AFDA@onconet.kiev.ua

Abbreviations used: 2'-5'A - 2',5'-oligoadenylate; BS-RNase bovine seminal RNase; HPC1 - hereditary prostate cancer; HPV - human papillomavirus; HVJ - hem-agglutinating virus of Japan; IFN - interferon; mRNA - messenger RNA; PNPase polynucleotide phosphorylase; RI - ribonuclease inhibitor protein; RIPs - ribosome-inactivating proteins; RNases - ribonucleases; siRNA - small interfering RNA; Topo - topoisomerase. polynucleotide phosphorylase (PNPase), RNase $\mathrm{PH}$, RNase II, RNase R, RNase D, RNase T, oligoribonuclease, exoribonuclease I and exoribonuclease II. These enzymes differently cleave various RNA species. Recently, researchers have paid attention to RNases as possible agents for the cancer treatment. The idea of using RNases for the treatment of cancer is to find an enzyme selectively damaging cancer cells without affecting the surrounding normal [4].

Today, several RNases allocated as possible candidates for anticancer agents [5-9]. The interferon (IFN) antiviral investigations and prostate cancer genetics have merged on a single-strand specific, regulated endoribonuclease. The $2^{\prime}, 5^{\prime}$-oligoadenylate $\left(2^{\prime}-5^{\prime} \mathrm{A}\right)$ system is an IFN-regulated RNA decay pathway that provides innate immunity against viral infections. The biologic action of the $2^{\prime}-5^{\prime} A$ system is mediated by RNase $L$, an endoribonuclease that becomes enzymatically active after binding to $2^{\prime}-5^{\prime} A$. RNase $L$ is also implicated in mediating apoptosis in response to both viral and nonviral inducers. To study the cellular effects of RNase $L$ activation, 2' $-5^{\prime} A$ was transfected into the human ovarian cancer cell line Hey1B. Activation of RNase $L$ by $2^{\prime}-5$ 'A resulted in specific $18 S$ rRNA cleavage and induction of apoptosis, as measured by TUNEL and annexin $\mathrm{V}$ binding assays. In contrast, the dimeric form of 2'-5'A, ppA2'p5'A, neither activated RNase $L$ nor caused apoptosis. Treatment with IFN-beta prior to 2' -5 'A transfection enhanced cellular RNase L levels 2.2-fold and increased the proportion of cells undergoing apoptosis (by 40\%). However, rRNA cleavages after 2'-5'A transfections were not enhanced by IFN-beta pretreatments, indicating that basal levels of RNase $L$ were sufficient for this activity. Apoptosis in response to RNase $L$ activation was accompanied by cytochrome $\mathrm{C}$ release from mitochondria. Induction of apoptosis by either 2'-5'A alone or by the combination of $2^{\prime}-5^{\prime} A$ and IFN-beta was effectively blocked with either the pancaspase inhibitor, Z-VAD-fmk, or with the caspase 3 inhibitor, DEVDfmk. Therefore, activation of RNase $L$ by $2^{\prime}-5$ 'A leads to cytochrome $\mathrm{C}$ release into the cytoplasm and then to caspase activation and apoptosis. These results suggest potential uses for $2^{\prime}-5$ 'A in augmenting the anticancer activities of IFN [10]. 
The RNASEL gene, a strong candidate for the hereditary prostate cancer 1 (HPC1) allele, encodes a single-stranded specific endoribonuclease involved in the antiviral actions of IFNs. Xiang et al. [11] have shown that RNase $L$ is activated enzymatically after binding to unusual $5^{\prime}$-phosphorylated, 2', $5^{\prime}$-linked oligoadenylates $\left(2^{\prime}-5^{\prime} \mathrm{A}\right)$. Biostable phosphorothioate analogues of $2^{\prime}-5^{\prime} A$ were synthesized chemically and used to study the effects of naturally occurring mutations and polymorphisms in RNASEL. The 2'5'A analogues induced RNase $L$ activity and caused apoptosis in cultures of late-stage, metastatic human prostate cancer cell lines DU145, PC3, and LNCaP. However, DU145 and PC3 cells were more sensitive to $2^{\prime}-5^{\prime} \mathrm{A}$ than $\mathrm{LNCaP}$ cells, which are heterozygous for an inactivating deletion mutation in RNase L. The RNase activities of missense variants of human RNase $L$ were compared after expression in a mouse RNase $L(-/-)$ cell line. Several variants (G59S, I97L, I220V, G296V, S322F, Y529C, and D541E) produced similar levels of RNase L activity as wild-type enzyme. In contrast, the R462Q variant, previously implicated in up to $13 \%$ of unselected prostate cancer cases, bound 2' -5 'A at wild-type levels but had a 3-fold decrease in RNase activity. The deficiency in RNase Lactivity (R462Q) was correlated with a reduction in its ability to dimerize into a catalytically active connect. Furthermore, RNase L (R462Q) was deficient in causing apoptosis in response to $2^{\prime}-5^{\prime} \mathrm{A}$ consistent with its possible role in prostate cancer development. Authors support the notion that RNASEL mutations allow tumor cells to escape a potent apoptotic pathway [11].

Genetics studies from several laboratories in the U.S., Finland, and Israel, support the recent identification of the RNase L gene, RNASEL, as a strong reason for the HPC1. Results from these studies suggest that mutations in RNASEL predispose men to an increased incidence of prostate cancer, which in some cases reflect more aggressive disease and/or decreased age of onset compared with nonRNASEL linked cases. RNase $L$ is a uniquely regulated endoribonuclease that requires $5^{\prime}$-triphosphorylated, $2^{\prime}, 5^{\prime}$-linked oligoadenylates $\left(2^{\prime}-5^{\prime} A\right)$ for its activity. The presence of both germline mutations in RNASEL segregating with disease within HPC-affected families and loss of heterozygosity in tumor tissues suggest a novel role for the regulated endoribonuclease in the pathogenesis of prostate cancer. The association of mutations in RNASEL with prostate cancer cases further suggests a relationship between innate immunity and tumor suppression. It is proposed that RNase $L$ functions in counteracting prostate cancer by virtue of its ability to degrade RNA, thus initiating a cellular stress response that leads to apoptosis [12, 13]. The HPC1 allele maps to the RNASEL gene encoding a protein (RNase $L$ ) implicated in the antiviral activity of IFNs.

To investigate the possible role of RNase L in apoptosis of prostate cancer cells, Malathi et al. [14] decreased levels of RNase $L$ by several fold in the
DU145 human prostate cancer cell line through the stable expression of a small interfering RNA (siRNA). Control cells expressed siRNA with three mismatched nucleotides to the RNase $L$ sequence. Cells deficient in RNase L, but not the control cells, were highly resistant to apoptosis by the RNase L activator, 2'5'A. At the same time, the RNase L-deficient cells were also highly resistant to apoptosis by combination treatments with a topoisomerase (Topo) I inhibitor (topotecan) and tumor necrosis factor-related apoptosis-inducing ligand TRAIL (Apo2L). In contrast, cells expressing siRNA to the RNase L inhibitor RLI (HP68) showed enhanced apoptosis in response to Topo I inhibitor alone or in combination with TRAIL. An inhibitor of c-Jun $\mathrm{NH}(2)$-terminal kinases reduced apoptosis induced by treatment with either $2^{\prime}-5^{\prime} \mathrm{A}$ or the combination of camptothecin and TRAIL, thus implicating c-Jun $\mathrm{NH}(2)$-terminal kinase in the apoptotic signaling pathway. Likewise, prostate cancer cells were sensitive to apoptosis from the combination of $2^{\prime}-5^{\prime} A$ with either TRAIL or Topo I inhibitor, whereas normal prostate epithelial cells were partially resistant to apoptosis. These findings indicate that RNase $L$ integrates and amplifies apoptotic signals generated during treatment of prostate cancer cells with $2^{\prime}-5^{\prime} \mathrm{A}$, Topo I inhibitors, and TRAIL [14].

Montia et al. [15] found that an extracellular RNase is involved in the control of ovarian tumorigenesis. They shown that the loss of function of RNase T2, an ancient and phylogenetically conserved RNase, plays a crucial role in ovarian tumorigenesis.

The above data were the basis for many attempts to use RNases for cancer therapy. Currently, it is shown that the RNases isolated from different sources, have antitumour activity. In some cases, studies reported to the clinical trials.

Bovine seminal RNase (BS-RNase). The RNase found in bull semen, displays antitumor, antispermatogenic, and immunosuppressive activities. Besides its unique structure and enzymatic properties seminal RNase belongs to an interesting group of RNases, the RISBASES (RNases with Special, Biological Actions), other members of which include angiogenin, selectively neurotoxic RNases, a lectin and the incompatibility factors from a flowering plant [16].

BS-RNases, a dimeric protein found to be homogeneous by several standard criteria of purity, is heterogeneous when analyzed by ion-exchange chromatography on carboxymethylcellulose. Three increasingly cationic subforms can be separated. The heterogeneity is due to the presence of two types of subunits, alpha and beta, which make up three isoenzymic dimers: alpha 2, beta 2, and alpha beta. Deamidation reactions can convert the most cationic beta 2 subform into the alpha beta subform, which in turn can be converted into stable alpha 2 subform. These conversions involve the hydrolysis of $2 \mathrm{~mol}$ of differentially labile amide groups per mol of protein. The ratios alpha 2: alpha beta: beta 2 are constant in all preparations of seminal RNase tested; they are independent of the purification 
procedure as well as of the biological source of the enzyme (seminal plasma or seminal vesicles). These results indicate that deamidations occur in vivo before the protein is secreted from the seminal glands. They also suggest that heterogeneity of seminal RNase reflects a physiological need of distinct molecular forms of enzyme or, alternatively, a process which leads to the aging of the protein [17]. The cytotoxic effect of BS-RNase on tumor cells is accompanied by the induction of apoptosis [17].

Sinatra et al. [18] provide ultrastructural and flow cytometry evidence of apoptotic death following BSRNase treatment, in normal cells and phytohemagglutinin-stimulated lymphocytes. Transmission and scanning electron microscopy, which were fully supported by flow cytometry data, showed typical features of apoptosis, such as decreased cell size, chromatin condensation, fragmentation in micronuclei, and the presence of apoptotic bodies. BS-RNase is a homologue of bovine pancreatic ribonuclease (RNase A).

Unlike RNase A, BS-RNase has notable toxicity for human tumor cells. Wild-type BS-RNase is a homodimer linked by two intermolecular disulfide bonds. This quaternary structure endows BS-RNase with resistance to inhibition by the cytosolic ribonuclease inhibitor protein (RI), which binds to RNase A and monomeric BS-RNase. Authors report on the creation and analysis of monomeric variants of BS-RNase that evade RI but retain full enzymatic activity [19]. The cytotoxic activity of these monomeric variants exceeds that of the wildtype dimer by up to 30-fold, indicating that the dimeric structure of BS-RNase is not required for cytotoxicity. Dimers of these monomeric variants are more cytotoxic than wild-type BS-RNase, suggesting that the cytotoxicity of the wild-type enzyme is limited by $\mathrm{RI}$ inhibition following dissociation of the dimer in the reducing environment of the cytosol. Finally, the cytotoxic activity of these dimers is less than that of the constituent monomers, indicating that their quaternary structure is a liability. These data provide new insight into structure - function relationships of BS-RNase. Moreover, BS-RNase monomers described herein are more toxic to human tumor cells than is any known variant or homologue of RNase A including ranpirnase, an amphibian homologue in phase III clinical trials for the treatment of unresectable malignant mesothelioma [20].

Onconase (ranpirnase) is a first-in-class therapeutic product based on Alfacell's proprietary RNase technology. A natural protein isolated from the leopard frog Rana pipiens, ranpirnase has been shown in the laboratory and clinic to target cancer cells while sparing normal cells. Ranpirnase triggers apoptosis, the natural death of cells, via multiple molecular mechanisms of action [21]. Ranpirnase is a novel RNase which preferentially degrades tRNA, thus leading to inhibition of protein synthesis and, ultimately, to cytostasis and cytotoxicity. Ranpirnase has demonstrated antitumor activity both in vitro and in vivo in several tumor models [22, 23]. The maximum tolerated dose emerging from phase I studies was $960 \mathrm{~g} / \mathrm{m}^{2}$, with renal toxicity as the main dose-limiting toxicity. A large phase II trial showed that ranpirnase has disease-modifying activity against malignant mesothelioma. Ranpirnase proved to be general caspase inhibitors strongly suggest that the signaling pathways triggered by the death stimuli diverge into one pathway leading to caspase activation (PARP processing, DNA degradation, and annexin $\mathrm{V}$ binding) and another leading to mRNA degradation. In addition, data obtained show that extensive mRNA degradation, although apparently a general early apoptotic event, is not sufficient to induce cell death per se. The molecular mechanisms implicated in apoptosis-induced mRNA degradation are not known. The apoptotic stimuli leads to activation of an RNase dependent on an upstream apical caspase (inhibited by Z-VAD-fmk, but not p35, and unable to cleave PARP) in a similar manner as CAD (caspase-activated DNase) activation. Conversely, activation of the RNase may be independent of caspase activation but still inhibited by Z-VAD-fmk [24]. Ranpirnase has been granted fast track status and orphan-drug designation for the treatment of malignant mesothelioma by the Food and Drug Administration. Additionally, ranpirnase has been granted orphan-drug designation in the European Union and Australia.

In April, 2006 Alfacell released interim data from the company's ongoing Phase IIIb randomized clinical trial of ranpirnase and doxorubicin for the treatment of malignant mesothelioma. The study reached the first interim analysis at 105 events (patient deaths) of the total 316 patients enrolled. Interim data demonstrate that the overall median survival time favored the ranpirnase plus doxorubicin treatment group (12 months) over the doxorubicin group (10 months). It has been tested and found to be cytotoxic to cancer cells because of its enzymatic activity against RNA.

Ranpirnase is internalized by endocytosis and released into the cytosol of the cancerous cell, where it selectively degrades tRNA beyond repair. In doing so, ranpirnase inhibits protein synthesis, stops cell cycle proliferation, and induces apoptosis [25].

USA-based Tamir Biotechnology (formerly Alfacell) says that scientists supported by the National Institute of Allergy and Infectious Diseases (NIAID) reported test results confirming two of its lead compounds showed excellent in vitro antiviral activity and no cellular toxicity at dose levels tested for human papillomavirus (HPV). Testing was performed using the HPV 11 strain, which along with HPV type 6, is responsible for $90 \%$ of genital or anal warts [26].

Ranpirnase is a cytotoxic RNase which targets tumor cells in vivo and in vitro. To date, cellular tRNA appeared to be the major target for ranpirnase mediated cytotoxic activity. Saxena et al. [27] demonstrated that ranpirnase can also cleave double-stranded RNA (dsRNA). Incubation of ranpirnase at $37^{\circ} \mathrm{C}$ with GAPDH gene-dsRNA ( 440 bp long) and dsRNA ladder showed degradation of dsRNA into a spectrum of smaller dsRNA fragments. Moreover, incubation of dsRNA substrates at $40^{\circ} \mathrm{C}$ under similar conditions 
markedly potentiated further cleavage of dsRNAs. The recently discovered double-stranded RNase activity of ranpirnase suggests another mechanism for inducing cell death/apoptosis in malignant phenotypes via the RNA interference mechanism involving siRNA and miRNA [27]. Another enzyme sialic acid-binding lectin (SBL), isolated from oocytes of Rana catesbeiana, is leczyme and has both lectin and RNase activities [8]. SBL agglutinates various kinds of tumor cells but not normal cells. SBL agglutination activity is not affected by mono- or oligosaccharides. However, SBL-induced agglutination and antitumor effects are inhibited by sialomucin but not asialomucin. SBL causes cancerselective induction of apoptosis by multiple signaling pathways, which target RNA [8].

RNase $\boldsymbol{T} \boldsymbol{1}$ is an endonuclease (EC 3.1.27.3), isolated from fungi, which cuts the single-stranded RNA molecules after guanine residues, i.e., at the $3^{\prime}$ end. RNase T1 is the best known representative of a large family of ribonucleolytic proteins secreted by fungi, mostly Aspergillus and Penicillium species. Ribotoxins stand out among them by their cytotoxic character. They exert their toxic action by first entering the cells and then cleaving a single phosphodiester bond located within a universally conserved sequence of the large rRNA gene, known as the sarcin - ricin loop. This cleavage leads to inhibition of protein biosynthesis, followed by cellular death by apoptosis. Although no protein receptor has been found for ribotoxins, they preferentially kill cells showing altered membrane permeability, such as those that are infected with virus or transformed.

The most-studied form of the enzyme RNase T1 is the mold of Aspergillus oryzae and Penicillium species. RNase $T 1$ is a small protein $\alpha+\beta$, consisting of 104 amino acid residues. Protein structure contains four antiparallel beta pleated sheets covered nearly five turnovers long alpha-helix. RNase T1 has two disulfide bonds Cys2-Cys 10 and Cys6-Cys 103, the latter of which is involved in the installation [28] and the full restoration of disulfide bonds leads to the unfolding of the protein [29].

Due to the specificity guanine RNase T1 frequently used for cleavage of RNA denatured prior to sequencing. Like other RNases, for example RNase A, RNase $\mathrm{T} 1$ is a popular subject for study of protein folding [30].

So, ranpirnase and $\alpha$-sarcin are known to be toxic to tumor cells, and on the other hand, their structure is related to that of RNase T1, latter is noncytotoxic because of its inability to internalize into tumor cells. In their study, Yuki et al. [31] internalized RNase T1 into human tumor cells via a novel gene transfer reagent, hem-agglutinating virus of Japan (HVJ) envelope vector, which resulted in cell death. This cytotoxicity was drastically increased by pretreatment of HVJ envelope vector with protamine sulfate, and was stronger than that of ranpirnase, which is in phase III human clinical trials as a nonmutagenic cancer chemotherapeutic agent. Furthermore, internalized RNase T1 induced apoptotic cell death programs. Because its cytotoxic- ity is unfortunately not specificttotumooceded| idcidrannot at present be developed as an anticancer drug. However, authors believe that RNase T1 incorporated in HVJ envelope vector will be a unique anticancer drug if HVJ envelope vector can be targeted to tumor cells [28, 32].

Specific complexes of protein and RNA carry out many essential biological functions, including RNA processing, RNA turnover, RNA folding, as well as the translation of genetic information from mRNA into protein sequences. Messenger RNA ( $m R N A$ ) decay is now emerging as an important control point and a major contributor to gene expression. Continuing identification of the protein factors and cofactors, and mRNA instability elements responsible for mRNA decay allow researchers to build a picture of the special processes involved in mRNA decay and its regulation [33].

Presently known data demonstrate that apoptosisinduced $\mathrm{mRNA}$ degradation is an early event triggered by different apoptotic signals, occurring not only in in vitro models but also in vivo. It is not restricted to genes with a specific function, since besides 28S rRNA, it affects mRNA coding for proteins implicated in a variety of functions, including cell type-specific functions such as HLA-I, IAa, TCR 3 , and CD69, structural functions such as $\beta$-actin, or control of cell survival, such as BAX. There is evidence that potassium channels, the NF-KB signaling pathway, and various caspases play a role in exogenous RNase-induced apoptosis [9].

Apoptosis-induced mRNA degradation is an active process that induces a similar decay kinetics ( $\mathrm{t} 1 / 2$ between 1.5 and $3 \mathrm{~h}$ ) of $\mathrm{mRNAs}$ with very different intrinsic half-lives (from 20 min to $>10 \mathrm{~h}$ ). Kinetic experiments using stimuli diverge into one pathway leading to caspase activation (PARP processing, DNA degradation, and annexin $\mathrm{V}$ binding) and another leading to mRNA degradation were held. In addition, the data obtained show that extensive mRNA degradation, although apparently a general early apoptotic event, is not sufficient to induce cell death per se. The molecular mechanisms implicated in apoptosis-induced mRNA degradation are not known. Nonetheless, it seems that the apoptotic stimuli leads to activation of an RNase dependent on an upstream apical caspase (inhibited by Z-VAD-fmk, but not $\mathrm{p} 35$, and unable to cleave PARP) in a similar manner as CAD activation. Conversely, activation of the RNase may be independent of caspase activation but still inhibited by Z-VAD-fmk [34].

Actibind and RNase T2. Actibind, a protein that is produced by the black molds Aspergillus niger, a well-known microorganism used in bio and food industry [32]. In plants, actibind binds actin, a major component of the intracellular structure in plants, interfering with the plants' pollen tubes and halting cell growth. Actibind can also affect mammalian cancer development [35]. RNase T2, was also subsequently found to bind actin in human and animal migrating cells, such as the cells that are responsible for new blood vessel formation (angiogenesis) in tumors thus blocking the blood supply to the tumors, actibind 
halted the ability of malignant cells to move through the blood stream to form new metastases [36].

A further plus is that actibind is not toxic to normal cells, thereby significantly minimizing the risk of side effects. The fungal actibind and the human RNase T2 represent the basis for a new class of drugs that could be used as a front-line therapy in the fight against cancer.

a-Sarcin. a-Sarcin, mitogillin, and restrictocin are small (approximately $17 \mathrm{kDa}$ ) basic ribosomeinactivating proteins (RIPs) produced by the Aspergilli that catalytically inactivate the large ribosomal subunits of all organisms tested to date. These three fungal ribotoxins act as specific RNases by hydrolyzing one single phosphodiester bond in the universally conserved alpha-sarcin domain of 23-28S rRNAs and are among the most potent inhibitors of protein synthesis known.

Previous molecular studies of ribotoxins indicated that they belong to the superfamily of RNases and analysis of the mitogillin gene employing PCR-mediated site-specific mutagenesis suggests that certain domains in ribotoxins, which share homologies with motifs in ribosome-related proteins, may be responsible for the targeting of ribotoxins to the ribosome. The applications of the ribotoxins as tools in research and their using as therapeutic and diagnostic agents are reviewed [37]. a-Sarcin is a potent polypeptide toxin (cyclising RNase) of 150 residues secreted by the fungus Aspergillus giganteus MDH18894 that belongs to the type 1 (those having only single polypeptide chain) group of the ribosome-inactivating enzyme. It is the most significant member of the family of fungal ribotoxins that display a 3-dimensional structure.

His50, Glu96, His 137 residues of a-sarcin involved in the mechanism of catalysis. The hydrolysis of $3^{\prime}-5^{\prime}$ phosphodiester bond of the substrate yielding 2 ' -3 ' cyclic mononucleotide and then conversion of the intermediate into the corresponding $3^{\prime}$-monophosphate derivative as the final product of the reaction. Thus, $\alpha$-sarcin acts by cleavage of the phosphodiester bond of 28S rRNA, stops the protein synthesis. In addition to this enzymatic activity $\alpha$-sarcin interacts with the lipid bilayers promoting their fusion and leakage. Any toxin that is able to produce more than $90 \%$ of inhibition of protein synthesis may induce apoptosis [38, 39].

a-Sarcin is active against transformed or virusinfected mammalian cells, in the absence of any other permeabilizing agent $[40,41]$. RNases distinct from ubiquitin-like peptides and proteins were isolated from several mushroom species. One of RNases was isolated from Pleurotus sajor-caju; it exerts an antiproliferative action on hepatoma and leukemia cells, and anti-mitogenic action on mouse spleen cells [42]. Recently was shown that antiproliferative activity can be attributed to enzymes which inactivate ribosomes by eliminating one or more adenosine residues from rRNA. During the past decade, mushroom inactivating proteins were isolated from several species including Calvatia caelata, F. velutipes, $H$. marmoreus, Lyophyllum shimeiji, and Pleurotus tuber-1 ribosome [43, 44].
RNase $\boldsymbol{P}$. RNase $\mathrm{P}$ is unique from other RNases in that it is the ribozyme, i.e. RNA that acts as a catalyst in the same way that the protein based enzyme would. Its function is to cleave off the extra or precursor sequence of RNA on RNA molecules. In the current cancer therapy, the main problem is to distinguish between the cancer cells and the normal cells. There are certain chimeric molecules, which are specific to the cancer cells, which can act as the specific targets and thus solving the problem [45]. M1 RNA is the catalytic subunit of RNase $P$ and this subunit catalyses the hydrolytic removal of $5^{\prime}$-leader sequence of t-RNA. Studies of the substrate recognition by M1 RNA have led to the development of the strategy of gene targeting by M1 RNA [45]. M1 RNA can be targeted to the mRNA simply by the addition of the so-called guide sequence at the $3^{\prime}$-terminal. So this now becomes as M1-GS, which has mRNA as its target. Cleavage of the mRNA will not allow the formation of the fusion proteins, which are specific for the cancer cells. The utility of the M1GS in the cancer was shown by its use against $B C R-A B L$ oncogene model [45]. This $B C R-A B L$ oncogene was created by the translocation of the sequences from $A B L$ gene on chromosome 9 to the $B C R$ gene on the chromosome 22 [46]. Two oncogenes were created $B C R-A B L \mathrm{p} 190$ and $B C R-A B L \mathrm{p} 210$. Both of these differ and have identical $A B L$ derived sequences but differ in the number of the $B C R$ nucleotides. These chimeric molecules so formed by the chromosomal rearrangement would be specific to the cancer cells and thus serve as the excellent targets. These $B C R$ $A B L$ oncogenes are responsible for the myelogenous leukemia and acute lymphoblastic leukemia [47]. BCR$A B L$ oncogenes inhibit the apoptosis by $\mathrm{Bcl}-2$ pathway as a part of their oncogenic phenotype. Inhibition of the $B C R-A B L$ expression would thus reverse this phenotype and the cells die by apoptosis. It should be noted that M1-GS should target only at the junction sequences of the transcribed mRNA. If not so then the mRNA of the normal cells would be cleaved and thus the resultant damage to the normal cells would occur. This gene therapy promises to be an effective strategy for the future treatment of the cancer.

Using only this $B C R-A B L$ system, model has created the RNase P technology for the inhibition of the chimeric gene products and the efficiency of the agents has not been evaluated in the animal models to our knowledge and thus the efficiency of the delivery process is still a major problem to be investigated and solved. But surely this advancement provides a new therapeutic tool for the treatment of cancer and holds some promise for more selective, non-toxic cancer therapy in the future.

These data show that the sources of bioactive RNases may be of different origin. It is known that a huge amount of unexplored RNases contain plants. Vegetation, as well as the world of fungi, presents a potentially inexhaustible source of these enzymes. It can be expected that some of these will possess anti-tumor activity. From the above it is clear that fur- 
ther study of antitumor action of RNases is possible in several directions.

1. The search for new sources of the enzymes with the new physiological properties. In this regard, we can point to such a source as the plants and fungi. It is known that the flora is characterized by an exceptionally large variety of RNases. It can be expected that some of these will possess anti-tumor activity. An important source of the enzymes can also be mushrooms. Well-known antitumor activity of many fungi at least partially may be explained by their enzymatic activity.

2. The study must be focused on the search for new inducers of RNases activity in the tissues, including tumor tissue. The same way the latent RNase $L$ is activated by $2^{\prime}-5^{\prime} A$ which in turn is synthesized under the influence of IFN.

3. Chemical modification of known RNases to enhance their resistance to intracellular inhibitors.

4. Creation of new ribozyme-type highly specific RNases aimed to the precise destruction of the products of oncogenes.

5. Perspective can be also combined use of RNases with known anticancer drugs. We believe that further development of these studies will be a prerequisite for the creation of new effective agents for the treatment of cancer.

\section{REFERENCES}

1. Iordanov MS, Ryabinina OP, Wong J, et al. Molecular determinants of apoptosis induced by the cytotoxic ribonuclease Onconase: evidence for cytotoxic mechanisms different from inhibition of protein synthesis. Cancer Res 2000; 60: 1983-94.

2. Kim JS, Soucek J, Matousek J, et al. Mechanism of ribonuclease cytotoxicity. Am Soc Biochem Mol Biol 1995; 270: $1097-102$.

3. Mi Y, Yu Q, Min Z. The meta-analysis results showed evidence that RNASEL Arg462Gln and Asp541Glu polymorphisms are associated with prostate cancer risk and could be low-penetrance prostate cancer susceptibility biomarkers. J Biomed Res 2010; 24: 365-73.

4. Benito A, Riba M, Vilanova M. On the track of antitumour ribonucleases. Mol Biosyst 2005; 1: 294-302.

5. Rutkoski TJ, Kurten EL, Mitchell JC, et al. Disruption of shape-complementarity markers to create cytotoxic variants of ribonuclease. J Mol Biol 2005; 354: 41-54.

6. Glinka EM. Killing of cancer cells through the use of eukaryotic expression vectors harboring genes encoding nucleases and ribonuclease inhibitor. J Int Soc Oncodevelop Biol Med 2015; 36: 3147-57.

7. Mit'kevich VA, Makarov AA, Il'inskaia ON. Cell targets of antitumor ribonucleases. Mol Biol 2014; 48: 181-8.

8. Tatsuta T, Sugawara S, Takahashi K, et al. Leczyme: a new candidate drug for cancer therapy. Bio Med Res Intern 2014; ID421415 (http://dx.doi.org/10.1155/2014/421415).

9. Castro J, Ribó M, Benito A, Vilanova M. Minireview: nucleus-targeted ribonucleases as antitumor drugs. Curr Med Chem 2013; 20: 1225-31.

10. Rusch L, Zhou A, Silverman RH. Caspase-dependent apoptosis by 2',5'-oligoadenylate activation of RNase L is enhanced by IFN-beta. J Interferon Cytokine Res 2000; 20: 1091-100.

11. Xiang Y, Wang Z, Murakami J, et al. Effects of RNase $\mathrm{L}$ mutations associated with prostate cancer on apopto- sis induced by 2',5'-oligoadenylates. Cancer Res 2003; 15: 6795-801.

12. Silverman RH. Implications for $\mathrm{RNase} L$ in prostate cancer biology. Biochemistry 2003; 42: 1805-12.

13. Olson KA, Byers HR, Key ME, et al. Prevention of human prostate tumor metastasis in athymic mice by antisense targeting of human angiogenin. Clin Cancer Res 2001; 7: 3598-605.

14. Malathi K, Paranjape JM, Ganapathi R, et al. HPC1/ RNASEL mediates apoptosis of prostate cancer cells treated with 2',5'-oligoadenylates, topoisomerase I inhibitors, and tumor necrosis factor-related apoptosis-inducing ligand. Cancer Res 2004; 15: 9144-51.

15. Montia L, Turconia G, Fabbric M, et al. The loss of function of Ribonuclease T2, an ancient and phylogenetically conserved RNase, plays a crucial role in ovarian tumorigenesis. PNAS 2013; 14: 8140-5.

16. D'Alessio G, Di Donato A, Parente A, et al. Seminal RNase: a unique member of the ribonuclease superfamily. Trends Biochem Sci 1991; 16: 104-6.

17. di Donato A, D’Alessio G. Heterogeneity of bovine seminal ribonuclease. Biochemistry 1981; 20: 7232-7.

18. Sinatra F, Callari D, Viola $M$, et al. Bovine seminal RNase induces apoptosis in normal proliferating lymphocytes. Compar Biochem Physiol Part C: Toxicol Pharmacol 2001; 129: 175-91.

19. Antignani A, Naddeo M, Cubellis MV, et al. Antitumor action of seminal ribonuclease, its dimeric structure, and its resistance to the cytosolic ribonuclease inhibitor. Biochemistry 2001; 40: 3492-6.

20. Lee JE, Raines RT. Cytotoxicity of bovine seminal ribonuclease: monomer versus dimer. Biochemistry 2005; 44: $15760-7$.

21. Mosimann SC, Johns KL, Ardelt W, et al. Comparative molecular modeling and crystallization of $\mathrm{p} 30$ protein: a novel antitumor protein of Rana pipiens oocytes and early embryos. Proteins 1992; 14: 392-400.

22. Wu Y, Mikulski SM, Ardelt W, et al. A cytotoxic ribonuclease: study of the mechanism of Onconase cytotoxicity. J Biol Chem 1993; 268: 10686-93.

23. Wu YN, Saxena SK, Ardelt W, et al. A study of the intracellular routing of cytotoxic ribonucleases. Am Soc Biochem Mol Biol 1995; 270: 17476-81.

24. Porta C, Paglino C, Mutti L. Ranpirnase and its potential for the treatment of unresectable malignant mesothelioma. Biologics 2008; 2: 601-9.

25. Lee I, Lee YH, Mikulski SM, et al. Tumoricidal effects of Onconase on various tumors. J Surg Oncol 2000; 73: $164-71$.

26. Altomare DA, Rybak SM, Pei J, et al. Onconase responsive genes in human mesothelioma cells: implications for an RNA damaging therapeutic agent. BMC Cancer 2010; 10: $34-8$.

27. Saxena A, Saxena S K, Shogen K. Effect of Onconase on double-stranded RNA in vitro. Anticancer Res 2009; 29: 1067-71.

28. Yoshida H. The ribonuclease T1 family. Methods Enzymol 2001; 341: 28-41.

29. Maquat LE, Arraiano C. RNA Turnover in bacteria, archaea and organelles. Acad Press, 2008. 620 p.

30. Fabian H, Naumann D. Protein folding and misfolding: Shining light by infrared spectroscopy. Springer Science \& Business Media, 2011. 244 p.

31. Yuki S, Kondo Y, Kato F, et al. Noncytotoxic ribonuclease, RNase T1, induces tumor cell death via hemagglutinating virus of Japan envelope vector. Eur J Biochem 2004; 271: 3567-72. 
32. Carreras-Sangrà N, Alvarez-García E, HerreroGalán E, et al. The therapeutic potential of fungal ribotoxins. Curr Pharm Biotechnol 2008; 9: 153-60.

33. Maquat LE, Kiledjian M. RNA turnover in eukaryotes: Nucleases, pathways and analysis of mRNA decay. Academic Press, 2009. 656 p.

34. del Prete M J, Robles MS, Guio A. Degradation of cellular mRNA is a general early apoptosis-induced event. FASEB J 2002; 18: 10.1096/fj.02-0392fje.

35. Roiz L, Smirnoff $P$, Bar-Eli M, et al. Actibind, an actin-binding fungal T2-RNase with antiangiogenic and anticarcinogenic characteristics. Cancer 2006; 106: 2295-308.

36. Irie M, Ohgi K. Ribonuclease T2. Meth Enzymol 2001; 341: 42-55.

37. Kao R, Davies J. Fungal ribotoxins: a family of naturally engineered targeted toxins? Biochem Cell Biol 1995; 73: $1151-9$.

38. Olmo N, Turnay J, Buitrago GG, et al. Cytotoxic mechanism of the ribotoxin-sarcin induction of cell death via apoptosis. Eur J Biochem 2001; 268: 2113-23.

39. Carreras-Sangrà $\mathrm{N}$, Tomé-Amat $\mathrm{J}$, García-Ortega L, et al. Production and characterization of a colon cancer-specific immunotoxin based on the fungal ribotoxin $\alpha$-sarcin. Protein Eng Des Sel 2012; 25: 425-35.
40. Fernández-Puentes C, Carrasco L. Viral infection permeabilizes mammalian cells to protein toxins. Cell 1980; 20: 769-75.

41. Turnay J, Olmo N, Jimenez A, et al. Kinetic study of the cytotoxic effect of $\alpha$-sarcin, a ribosome inactivating protein from Aspergillus giganteus, on tumour cell lines: protein biosynthesis inhibition and cell binding. Mol Cell Biochem 1993; 122: 39-47.

42. $\mathrm{Ng}$ TB. Peptides and proteins from fungi. Peptides 2004; 25: 1055-73.

43. Xu X, Yan H, Chen J, et al. Bioactive proteins from mushrooms. Biotech Adv 2011; 29: 667-74.

44. Wasser SP, Weis AL. Therapeutic effects of substances occurring in higher Basidiomycetes mushrooms: a modern perspective. Crit Rev Immunol 1999; 19: 65-96.

45. Cobaleda IC, Garcia IS. In vivo inhibition by a sitespecific catalytic RNA subunit of RNase P designed against the BCR-ABL oncogenic products: a novel approach for cancer treatment. Blood 2000; 95: 731-7.

46. Garcia IS, Grutz G. Tumorigenic activity of the BCRABL oncogenes is mediated by BCL2. Proc Natl Acad Sci USA 1995; 92: 5287-91.

47. Heisterkamp N, Jenster G, Hoeve HT, et al. Acute leukemia in bcr/abl transgenic mice. Nature 1990; 344: 251-3. 